\title{
Syndrome de Papillon-Lefèvre : à propos d'un cas
}

\section{Papillon-Lefèvre syndrome : a case report}

\author{
MUSTAPHA EL ALLOUSSI ${ }^{1}$, DRISS BENNAZZA², EL MILOUD RAHMANI ${ }^{3}$, LAILA TOUFIKI ${ }^{4}$
}

\section{RÉSUMÉ}

Ce cas concerne une jeune fille âgée de 3 ans qui présente un syndrome de Papillon-Lefèvre et dont le diagnostic est basé sur les données cliniques. Elle présentait des hyperkératoses plantaires dès la première consultation avec une parodontite sévère et des dents mobiles ; les lésions palmaires ne sont apparues que quelques mois après la première consultation.

Le traitement initial a comporté la réalisation d'un traitement parodontal avec extractions des dents non conservables, puis la réalisation d'une prothèse dentaire pédiatrique. (Med Buccale Chir Buccale 2009 ; 15: 189 194).

mots clés : syndrome de Papillon-Lefèvre, hyperkératose, parodontite, traitement parodontal

\section{SUMMARY}

médecine buccale chirurgie buccale

VOL. $15, \mathrm{~N}^{\circ} 4$ 2009

page 189

A 3-year-old female presented with plantar skin lesions, tooth mobility, and advanced periodontitis. The palmars kin lesions are visible few month after first visit. Based on clinical findings, the cases were diagnosed with PLS. The initial treatment included treatment parodontal, avulsed the teeth mobile and to do a pediatric prosthetic. (Med Buccale Chir Buccale 2009 ; 15: 189-194).

key words: Papillon-Lefèvre syndrome, hyperkeratosis, periodontitis, periodontal treatment

1. Service d'Odontologie pédiatrique CCTD Rabat Faculté de Médecine dentaire Rabat

2. Service de Parodontologie CCTD Rabat Faculté de Médecine dentaire Rabat

3. Service de Prothèse adjointe CCTD Rabat Faculté de Médecine dentaire Rabat

4. Service de Prothèse conjointe CCTD Rabat Faculté de Médecine dentaire Rabat

Demande de tirés à part:

Mustapha El Aloussi Amal 0 immeuble 9 n¹ cité Yacoub El Mansour Rabat elalloussi99@yahoo.fr elalloussi@hotmail.com

Les 2 premiers auteurs ont participé à égalité à la rédaction de cet article. 
médecine
Le syndrome de Papillon-Lefèvre (SPL) est une maladie héréditaire autosomique récessive, rare, caractérisée par l'apparition vers l'âge de deux à trois ans d'une kératodermie palmoplantaire érythématosquameuse. La kératodermie palmoplantaire est une hyperkératose psoriasiforme, caractérisé par un épaississement de la peau de la paume des mains et/ou de la plante des pieds qui peut déborder sur la face dorsale des mains et des pieds. Des lésions psoriasiformes des membres peuvent être observées ${ }^{[3,10]}$.

Cette kératodermie s'associe dès la première enfance, à une parodontite sévère avec alvéolyse et chute prématurée des dents temporaires; dans la deuxième enfance, ce phénomène se reproduit avec une chute précoce des dents définitives. Le SPL s'accompagne dans la moitié des cas d'une susceptibilité aux infections (furonculose, abcès cutanés, hidrosadénites suppurées) ${ }^{[4]}$.

Ce rapport décrit, après une brève revue de la littérature, un cas dont le diagnostic et la prise en charge ont été réalisées par un médecin dentiste.

\section{OBSERVATION CLINIQUE}

L'enfant $A D$, âgée de 3 ans et demi, de sexe féminin, sans antécédents médico-chirurgicaux, s'est présentée en consultation accompagnée de ses parents pour des douleurs en rapport avec des dents très mobiles et pour la perte précoce de ses dents temporaires.

Les parents de la patiente qui étaient des cousins du premier degré rapportaient qu'il n'y avait aucun cas similaire dans la famille.

L'examen clinique a montré une hyperkératose plantaire, mais la paume des mains avait un aspect normal ; la mère signalait qu'elle avait observé la même lésion sur la paume des mains 2 mois auparavant mais les lésions avaient disparu spontanément (Fig. 1 et 2). Pendant le traitement, l'hyperkératose sur la paume des mains est réapparue (Fig. 3 et 4). L'examen endobuccal a montré une perte prématurée des dents temporaires, une inflammation gingivale sévère et généralisée, des récessions et des mobilités sur les dents résiduelles, signe de destruction parodontale sévère (Fig. 5 et 6 ).
L'examen radiographique (Fig. 7) a montré l'existence d'une lyse très marquée de l'os alvéolaire autour des dents résiduelles.

Un bilan complémentaire biologique comprenant la numération de la formule sanguine, un bilan biochimique ainsi qu'une radiographie du crâne étaient sans particularités.

Le diagnostic du SPL a été posé à partir des signes cliniques et radiographiques.

Le traitement a consisté dans un premier temps en l'instruction et l'enseignement à l'enfant des moyens et méthodes nécessaire pour l'hygiène bucco-dentaire, un détartrage et un surfaçage radiculaire avant de procéder à l'extraction des dents non conservables.

Dans un deuxième temps on a procédé à la réalisation d'une prothèse amovible afin de rétablir l'esthétique et les fonctions de mastication et de déglutition (Fig. 8 à 11).

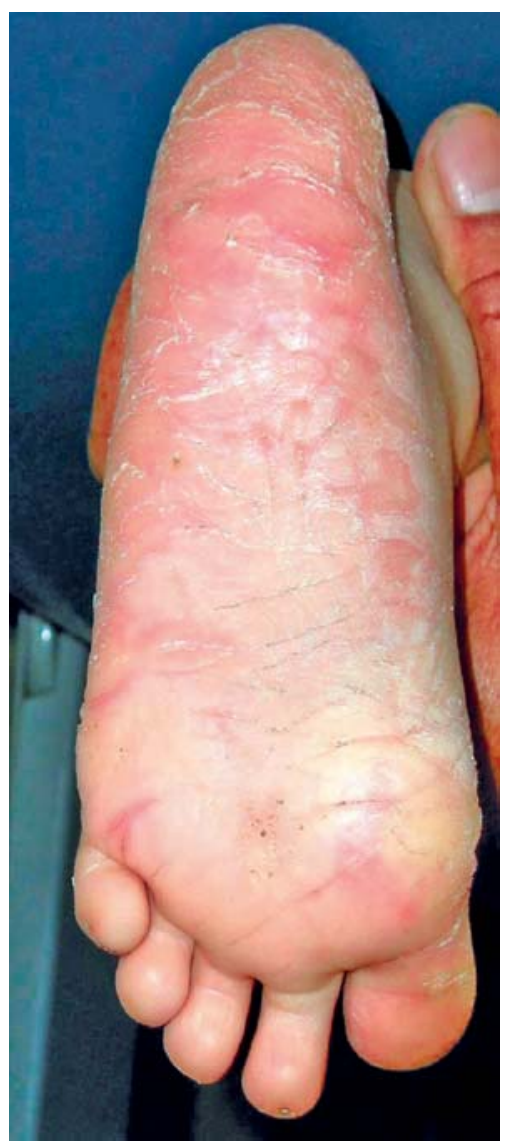

Figure 1 : Hyperkératose plantaire. Plantar hyperkeratosis. 


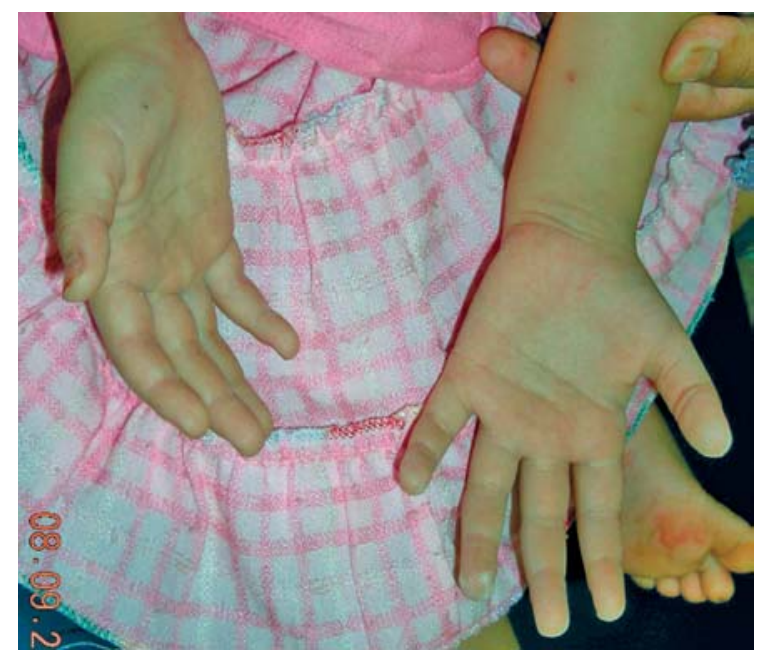

Figure 2 : Absence de lésion sur les mains. Hands without lesions.
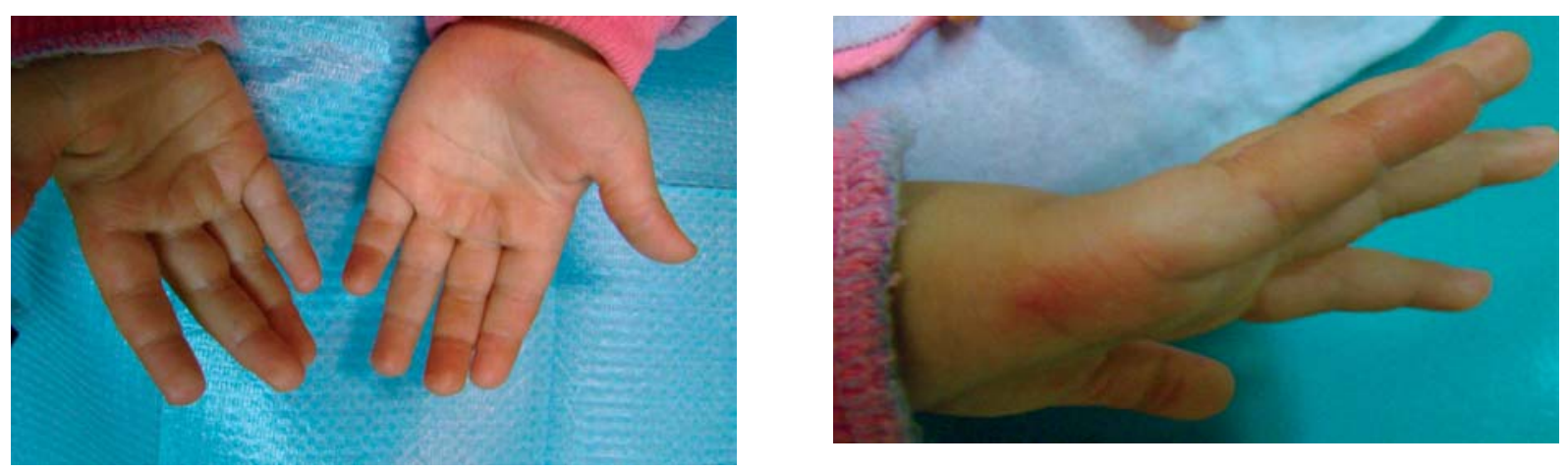

médecine

Figure 3-4 : Apparition d'une hyperkératose palmaire 2 mois plus tard. Apparition of a palmar hyperkeratosis two months later.
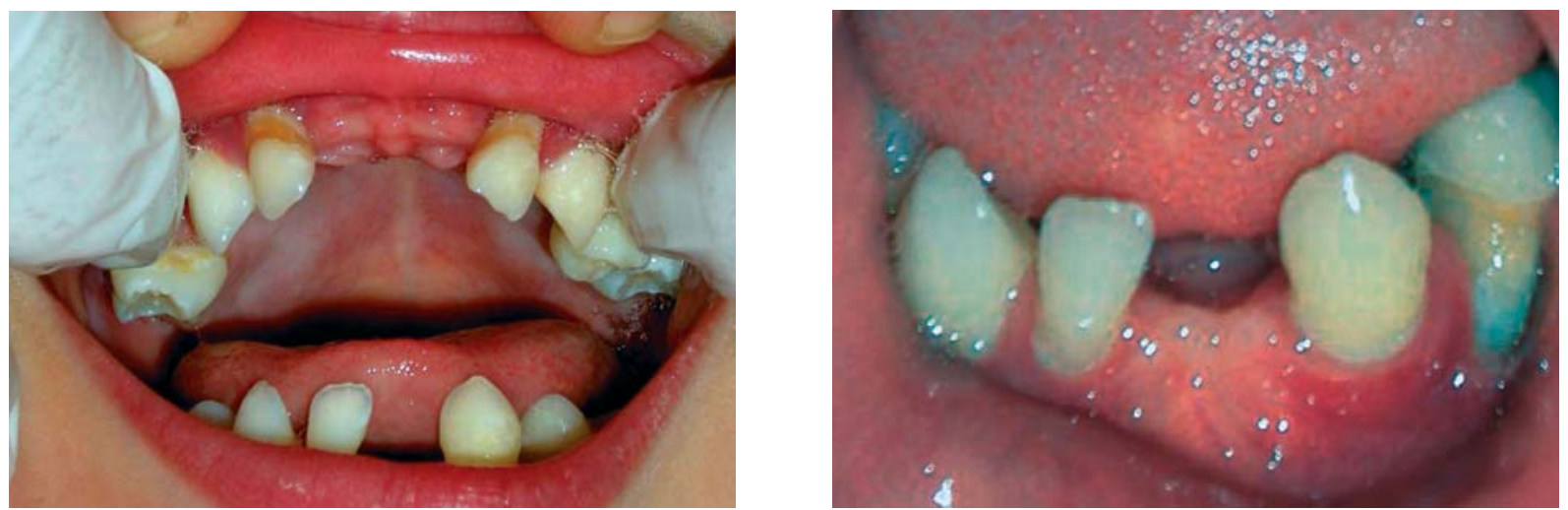

Figure 5-6 : Inflammation gingivale sévère avec présence des récessions gingivales et perte précoce des dents temporaires.

Severe gingival inflammation with gingival recession and early loss of decidual teeth. 


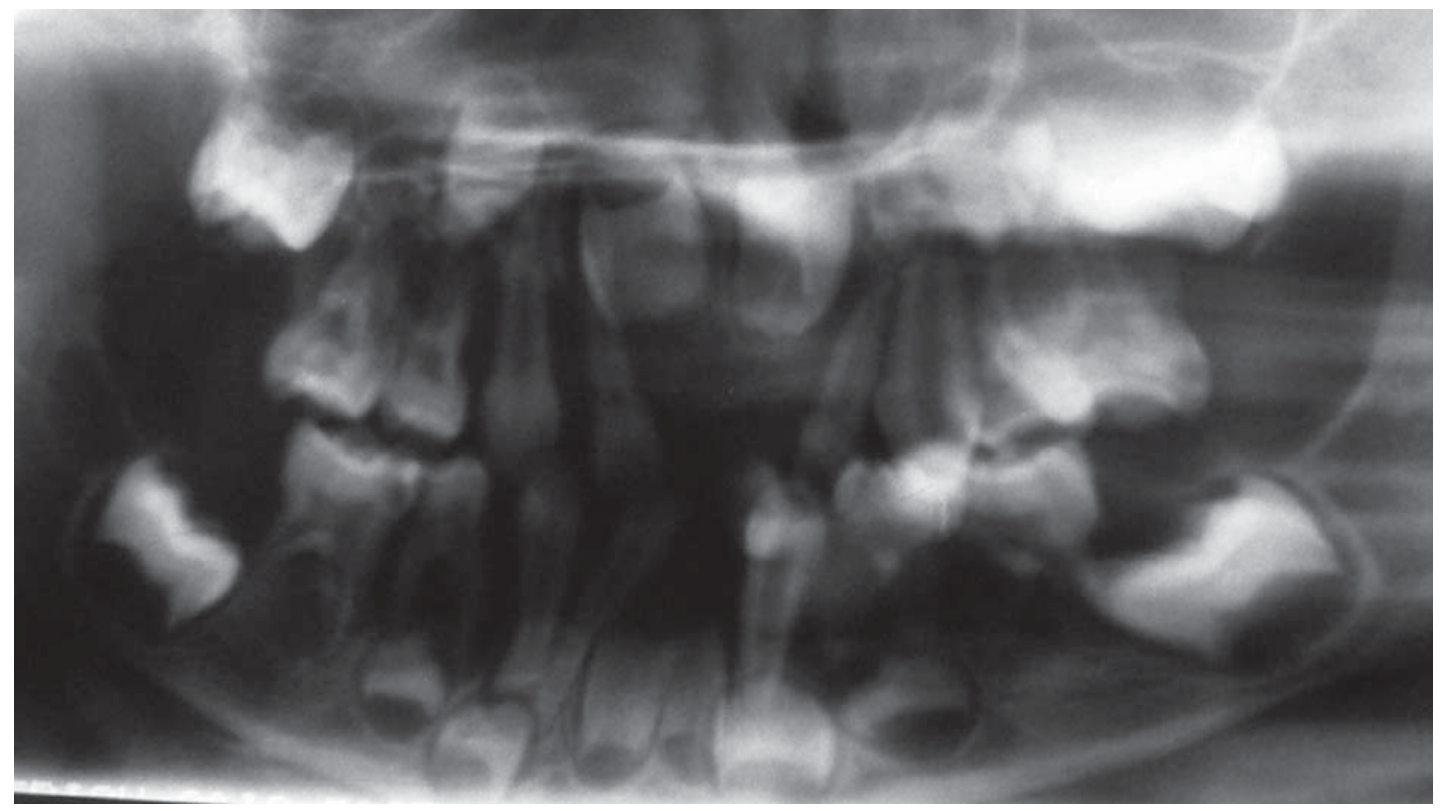

Figure 7 : Radiographie panoramique montrant une résorption importante de l'os alvéolaire des dents temporaires. Panoramic radiography showing an important resorption of the alveolar bone of eht decidual teeth.

médecine

buccale

chirurgie

buccale

VOL. $15, \mathrm{~N}^{\circ} 4$ 2009

page 192

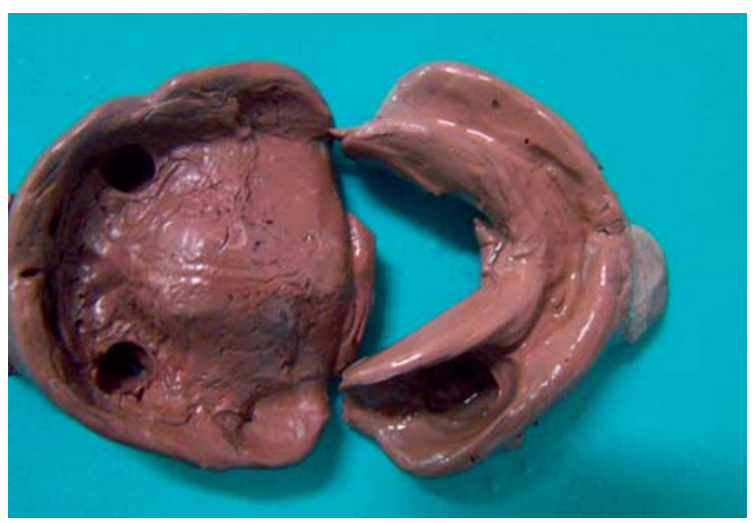

Figure 8 : Empreinte secondaire réalisée avec un porteempreinte individuel et du polusufine.

Secundary imprint with an individual imprint holder and polusufine.

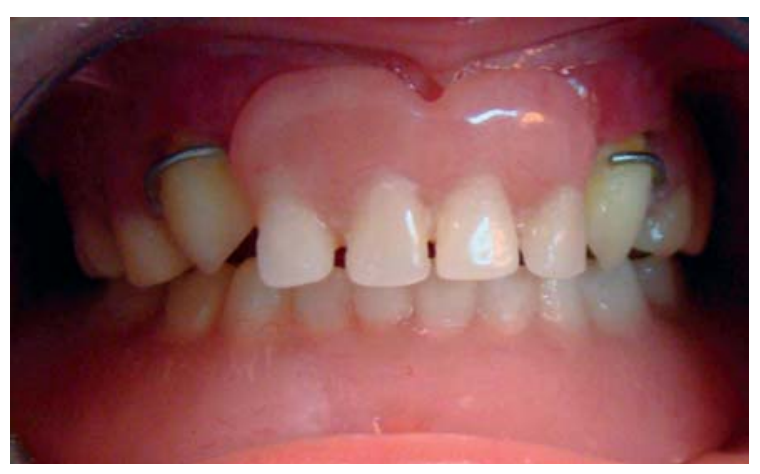

Figure 10 : Prothèse en bouche.

Prothesis in the mouth.

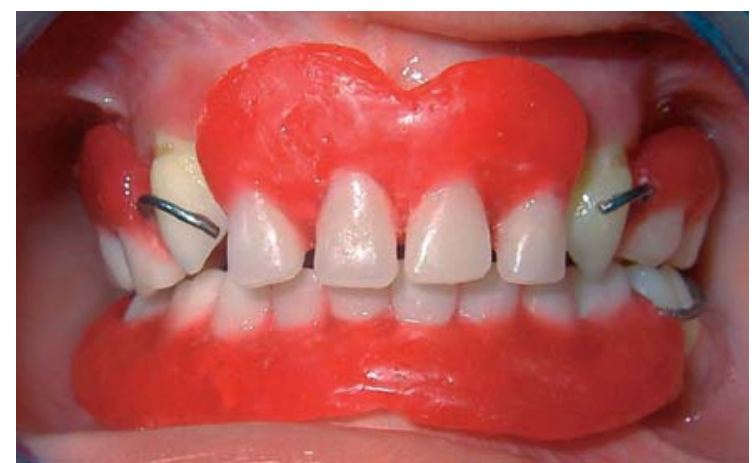

Figure 9 : Essayage de la prothèse en bouche. Test of the prothesis in the mouth.

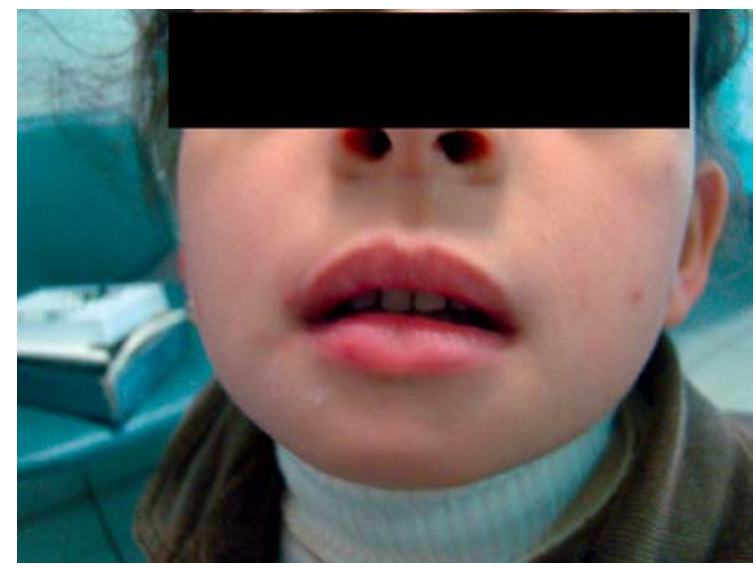

Figure 11 : Sourire de l'enfant avec la prothèse. Smile of the child with the prothesis. 


\section{DISCUSSION}

Ce type de kératose palmoplantaire a été décrit pour la première fois en 1924 par deux dermatologues français Papillon et Lefèvre. II s'agit d'une affection génotypique rare dont l'incidence est de 1 à 4 cas par million. Le SPL associe constamment des signes constants dermatologiques et dentaires ${ }^{[1,2]}$. II est caractérisé par une hyperkératose palmo-plantaire diffuse et érythémateuse. La kératodermie de type papillon-Lefèvre est nettement limitée par une bande de couleur rouge ; elle évolue par poussées et s'accompagne de rémissions partielles, quelquefois presque totales. Les lésions plantaires tendent à être plus sévères que les lésions palmaires ${ }^{[4]}$. Les manifestations apparaissant entre la $1^{\text {re }}$ et la $4^{\mathrm{e}}$ année de la vie ${ }^{[5]}$; vers l'âge de deux ans pour le cas rapporté. Les manifestations buccodentaires se caractérisent, après une éruption normale des dents temporaires, par l'apparition d'une inflammation gingivale qui s'accompagne d'une lyse de l'os alvéolaire, aboutissant à une exfoliation des dents temporaires vers l'âge de 4 à 5 ans, après la gencive retrouve un aspect normal. Les mêmes signes réapparaissent avec l'éruption des dents permanentes qui subissent la même évolution que les dents temporaires ${ }^{[1]}$. Comme pour plusieurs cas décrits dans la littérature le diagnostic a été posé sur les signes cliniques ${ }^{[3]}$.

Sur le plan génétique, la transmission est autosomique récessive et une consanguinité parentale est fréquemment retrouvée. II n'y a pas de prédilection selon le sexe. Le gène responsable du SPL qui a été localisé en 11q14/q21, code pour la cathepsine $\mathrm{C}$. Le SPL résulte d'une mutation du gène codant pour cette protéine donnant une suppression totale de son activité [8]. Cette protéine joue un rôle essentiel dans l'établissement et le maintien de l'organisation structurale de l'épithélium des extrémités (paumes, plantes), de même que dans l'intégrité des propriétés immunologiques des tissus de soutien des dents ${ }^{[8,11]}$. Concernant le cas présenté dans ce travail et après avis du généticien et du dermatologue, les signes cliniques et dermatologiques sont tellement évocateurs du syndrome que l'étude génétique n'a pas été réalisée.
Ce syndrome s'accompagne dans la moitié des cas d'une susceptibilité aux infections cutanées (furonculose, abcès cutanés) et d'une hidrosadénite suppurée [5,12]. Ces infections peuvent être expliquées par un état d'immunodépression lié à des anomalies du chimiotactisme et de la phagocytose et de l'adhérence des polynucléaires.

Le diagnostic différentiel comprend la maladie de Haim-Munk qui présente les mêmes signes cliniques dentaires et dermatologiques. Cependant, les lésions cutanées sont plus sévères alors que les lésions dentaires sont de moindre degré que dans le SPL.

La prise en charge des patients atteints du SPL nécessite une approche multidisciplinaire. Les manifestations cutanées sont généralement traitées avec des rétinoïdes et des kératolytiques. Les rétinoïdes oraux, tels que l'acitrétine, l'étrétinate et l'andisotretinoin auraient un effet bénéfique pour les lésions dentaires et cutanées. Le cas rapporté a bénéficié d'un traitement à base de rétinoïdes.

Le traitement de la parodontite agressive est pris en charge selon un protocole classique et comprend [12] :

- une phase de thérapeutique étiologique avec :

- l'enseignement des méthodes de brossage et motivation pour l'hygiène bucco-dentaire,

- un détartrage visant à éliminer les dépôts tartriques sus et sous-gingivaux,

- l'extraction des dents non conservables,

- un surfaçage radiculaire consistant en l'élimination des dépôts tartriques sus et sous-gingivaux résiduels, afin de réduire l'adhésion de la flore microbienne à la surface radiculaire,

- la prescription d'antiseptiques (bains de bouche à base de chlorhexidine) et d'une antibiothérapie (associant amoxicilline et métronidazole).

- la réévaluation

- une phase restauratrice consistant au remplacement prothétique des dents absentes par une prothèse provisoire visant à restaurer l'esthétique, la fonction et à maintenir le profil psychologique de l'enfant ;

- une phase de thérapeutique parodontale de soutien visant à maintenir les résultats obtenus et à contrôler l'évolution de la maladie. médecine

buccale

chirurgie buccale

VOL. $15, \mathrm{~N}^{\circ} 4$ 2009

page 193 


\section{CONCLUSION}

Le SPL est une maladie systémique qui s'accompagne d'une parodontite précoce et sévère. La prise en charge de cette maladie est lourde: est doit être multidisciplinaire et nécessite une étroite collaboration entre le dermatologue et le

\section{RÉFÉRENCES}

1 - Shahbaz AJ, Khachemoune A. Papillon-Lefèvre syndrome: case report and review of the literature. Dermatol Online J 10 (1): 13

2 - Peter H, Susanna K. Palmoplantar keratodermas. Clin Dermatol 2005 ; 23 : 15-22.

3 - Yagmur Bas A, Yilmaz G, Ertan U, Ikizoglu E, Ozkasap S, Karacan C. Papillon-Lefevre syndrome: A case report. Int Pediatr $2004 ; 19: 224-5$.

4 - Vikram K, Narbir ST, Nand LS. Papillon-Lefèvre syndrome. Indian Pediatr 2003 ; 40 : 1197-200.

médecine

buccale

chirurgie

buccale

VOL. $15, \mathrm{~N}^{\circ} 4$

2009

page 194
5 - Tinanoff N, Tempro P, Maderazo EG. Dental treatment of Papillon-Lefèvre syndrome: 15-year follow-up. J Clinl Periodontol 2005 ; 22 : 609-12.

6 - Cagli NA, Hakki SS, Dursun R, Toy H, Gokalp A, Ryu OH, Hart PS, Hart TC.Clinical, genetic, and biochemical findings in two siblings with Papillon-Lefèvre syndrome. J Periodontol 2005 ; 76 : 2322-9.

7 - Wara-Aswapati N, Lertsirivorakul J, Nagasawa T, Kawashima Y, Ishikawa I. Papillon-Lefèvre syndrome: serum immunoglobulin $\mathrm{G}$ (IgG) subclass antibody response to periodontopathic bacteria. A case report. J Periodontol $2001 ; 72$ : 1747-54. chirurgien dentiste. Ce dernier doit réaliser très précocement un traitement parodontal et réaliser une prothèse dentaire pédiatrique évolutive pour rétablir l'esthétique et la fonction.

Le chirurgien dentiste est fortement impliqué aussi bien pour le diagnostic que pour la prise en charge du SPL.

8 - Selvaraju V, Markandaya M, Prasad PV, Sathyan P, Sethuraman G, Srivastava SC, Thakker N, Kumar A. Mutation analysis of the cathepsin $\mathrm{C}$ gene in Indian families with Papillon-Lefèvre syndrome. BMC Med Genet $2003 ; 12: 4-5$.

9 - Eickholz P, Kugel B, Pohl S, Näher H, Staehle HJ. Combined mechanical and antibiotics periodontal therapy in a case of Papillon-Lefevre syndrome. J Periodontol $2001 ; 72$ : 542-7.

10 - Hart TC, Shapira L. Papillon-Lefèvre syndrome. Periodontol $20001994 ; 6$ : 88-100.

11 - Nitta N, Wara-Aswapati N, Lertsirivorakul J, Nakamura T, Yamamoto M. A novel mutation of the cathepsin $\mathrm{C}$ gene in a Thai family with Papillon-Lefèvre syndrome. J Periodontol 2005 ; 76 : 492-6.

12 - Ahuja V, Shin RH, Mudgil A, Nanda V, Schoor R. Papillon-Lefèvre syndrome: a successful outcome. J Periodontol 2005 ; 76 : 1996-2001 